\title{
Isolated gallbladder injury following blunt abdominal trauma: a case report
}

Received May 23, 2020

Revised June 8, 2020

Accepted June 9, 2020

Correspondence to

Kyoung Hoon Lim

Department of Surgery, Trauma

Center, Kyungpook National University

Hospital, 130 Dongdeok-ro, Jung-gu,

Daegu, 41944, Korea

Tel: +82-53-420-6527

Fax: +82-53-421-0510

E-mail:drlimkh@knu.ac.kr

\section{Kyoung Hoon Lim}

Department of Surgery, Trauma Center, Kyungpook National University Hospital, School of Medicine, Kyungpook National University, Daegu, Korea

Isolated gallbladder injury following blunt abdominal trauma is very rare. Early diagnosis of such injuries is often difficult due to their rarity and nonspecific symptoms. A surgeon's suspicion and knowledge about the characteristic imaging findings of gallbladder injury are important. This study presents a case of gallbladder injury following blunt abdominal trauma that was diagnosed by computed tomography.

Key Words: Gallbladder, Blunt injury, Computed tomography

\section{Introduction}

The gallbladder is located within the gallbladder fossa on the visceral surface of the right lobe of the liver and is sheltered by the right costal margin. Its anatomical location possibly accounts for the low incidence of gallbladder injuries following blunt abdominal trauma (1). The diagnosis of blunt injury to the gallbladder may often be difficult and delayed because of the nonspecific clinical symptoms. For early diagnosis, it is important to suspect gallbladder injury and understand the characteristic imaging findings. Here we present a case of gallbladder injury following blunt abdominal trauma that was diagnosed by computed tomography (CT).

\section{Case presentation}

A 45-year-old woman with a history of major depressive disorder was admitted to the emergency department after a fall from a height of 7 floors. She was alert and hemodynamically stable on arrival, and quadriparesis was observed. Physical examination of the abdomen did not yield reliable findings because of a cervical spinal cord injury. A whole-body scan was performed according to the trauma protocol, and C5-C6 dislocation, sternal fracture, multiple rib fractures with pneumothorax, and edematous gallbladder were revealed. The Injury Severity Score was 36. Abdominal contrast-enhanced CT showed an enlarged, edematous gallbladder, with active extravasation of intraluminal contrast medium and discontinuity of the mucosal layer (Fig. 1A, 1B). Scanty pericholecystic and perihepatic fluid was present.

Exploratory laparoscopy confirmed that the gallbladder was enlarged and edematous and had bile-stained contents (Fig. 2A). In addition, the taeniae coli outside the transverse colon were torn (Fig. 2B), and the mucosal and muscular layers were disrupted (Fig. 2C). Cholecystectomy and suture repair of the torn taeniae coli were successfully performed with laparoscopy. The patient's postoperative course was unremarkable.

(c)This is an Open Access article distributed under the terms of the Creative Commons Attribution Non-Commercial License (http://creativecommons.org/licenses/by-nc/4.0) which permits unrestricted noncommercial use, distribution, and reproduction in any medium, provided the original work is properly cited.

Copyright (C) 2020 Korean Association for Research, Procedures and Education on Trauma. All rights reserved. 


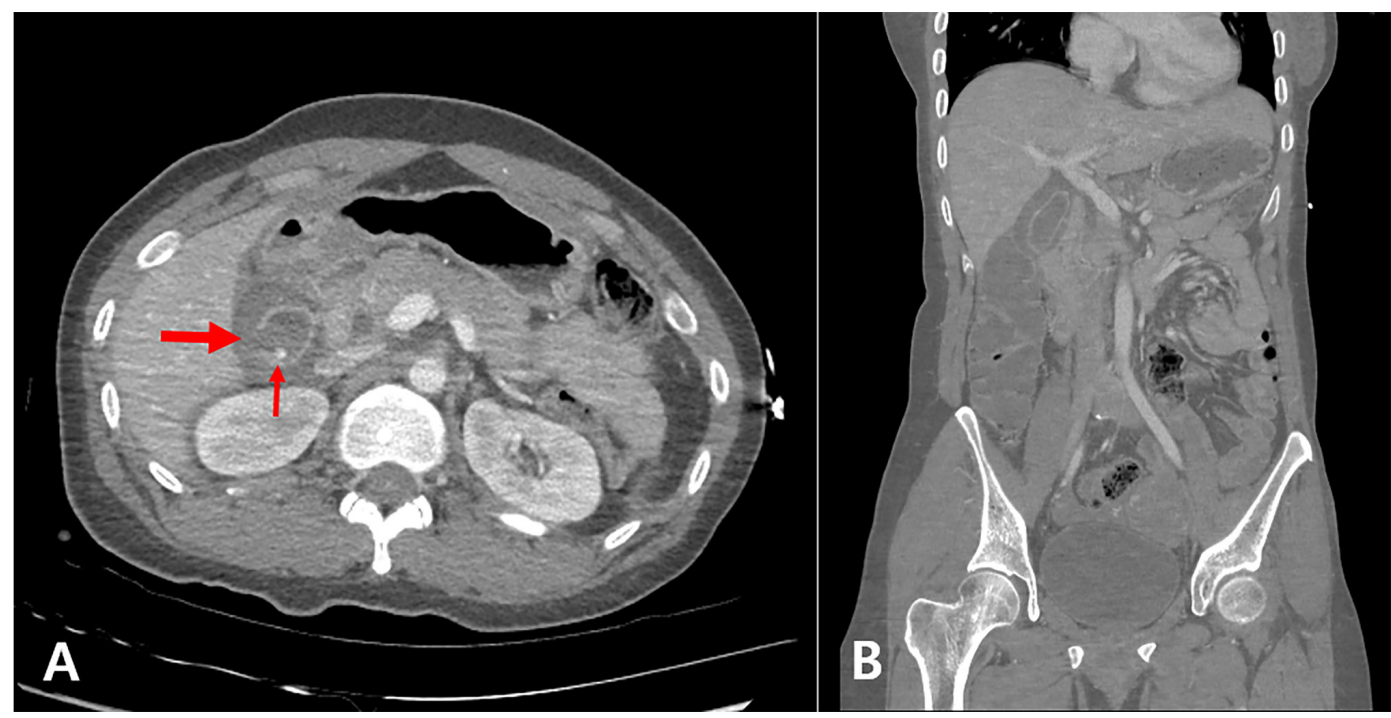

Fig. 1. Abdominal computed tomography revealed edema of the gallbladder wall, intraluminal extravasation of contrast medium (thin arrow), and discontinuity of the mucosal layer (thick arrow).

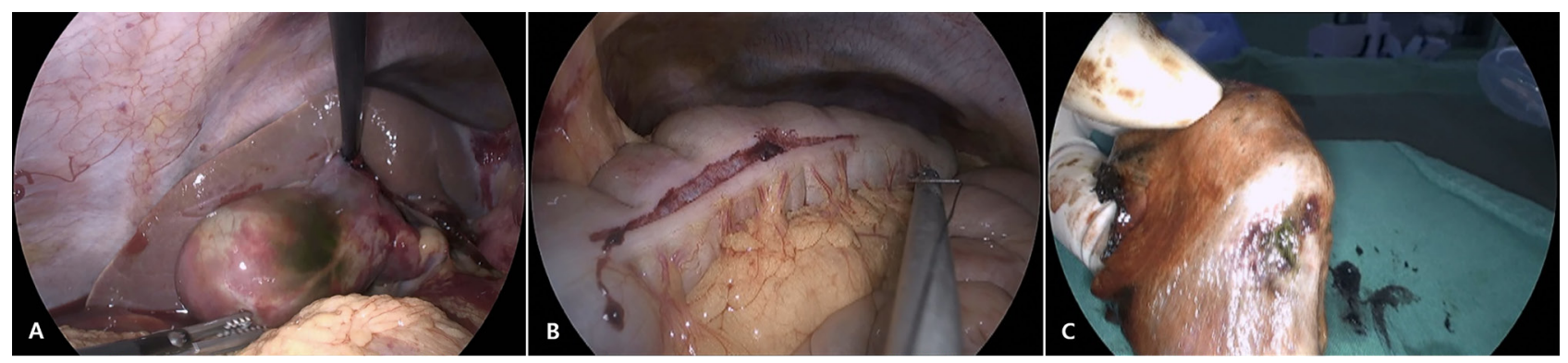

Fig. 2. Laparoscopic findings: (A) Edematous, bile-stained, and enlarged gallbladder; (B) Torn taeniae coli on the transverse colon; (C) Disrupted mucosal and muscular layers in gallbaldder..

\section{Discussion}

Gallbladder injury is rare and found in only approximately $2 \%$ of patients who undergo laparotomy for abdominal trauma (2). In the majority (89\%) of patients, gallbladder injury is caused by penetrating trauma, whereas gallbladder injury due to blunt abdominal trauma is rare (3). Blunt trauma is frequently associated with adjacent liver injury. Isolated gallbladder injury is very rare. Such an injury caused by blunt trauma is usually secondary to a direct blow or to shearing forces, which are more likely to cause an avulsion type of injury (2).

Isolated traumatic gallbladder injury is infrequent because of its anatomic location, given that the organ is protected by the adjacent liver and ribcage (1). Diagnosis of isolated gallbladder injury, especially contusion to the gall- bladder, is often delayed because of the lack of acute signs or symptoms, and such a delay can potentially increase the risk of morbidity $(1,2)$. Therefore, surgeons must consider gallbladder injury based on abnormal imaging findings.

$\mathrm{CT}$ is the most sensitive method for diagnosing gallbladder injury (4). The appearance of hyperdense blood within the gallbladder lumen is highly suggestive of gallbladder injury, and an irregularity of the gallbladder wall may be the only indication of an underlying gallbladder injury (4). The findings in our case were active hemorrhage within the gallbladder lumen and edematous wall thickening with discontinuity of the mucosal layer.

The management of gallbladder injury is based on clinical symptoms. Mild contusions may be treated conservatively. With perforation or avulsion, cholecystectomy is often the treatment of choice, and it yields favorable re- 
sults $(1,2)$. The mortality rate of patients with gallbladder injury is related to the associated injuries. The prognosis of patients with gallbladder injury in cases of early detection and lack of other severe associated injuries remains quite $\operatorname{good}(2,5)$.

\section{Conflict of interest}

No potential conflict of interest relevant to this article was reported.

\section{References}

1. Schachter P, Czerniak A, Shemesh E, Avigad I, Lotan $\mathrm{G}$, Wolfstein I. Isolated gallbladder rupture due to blunt abdominal trauma. HPB Surg. 1989;1(4):359-
62.

2. Soderstrom CA, Maekawa K, DuPriest RW Jr., Cowley RA. Gallbladder injuries resulting from blunt abdominal trauma: an experience and review. Ann Surg. 1981;193(1):60-6.

3. Ball CG, Dixon E, Kirkpatrick AW, Sutherland FR, Laupland KB, Feliciano DV. A decade of experience with injuries to the gallbladder. J Trauma Manag Outcomes. 2010;4(1):3.

4. Erb RE, Mirvis SE, Shanmuganathan K. Gallbladder injury secondary to blunt trauma: CT findings. J Comput Assist Tomogr. 1994;18(5):778-84.

5. Sharma O. Blunt gallbladder injuries: presentation of twenty-two cases with review of the literature. J Trauma. 1995;39(3):576-80. 